\title{
Application of $q$-Space Diffusion MRI for the Visualization of White Matter
}

\author{
Kanehiro Fujiyoshi, ${ }^{1,2,6 \star}$ Keigo Hikishima, ${ }^{2,7 \star}$ Jin Nakahara, ${ }^{3 \star}$ Osahiko Tsuji, ${ }^{1,2}$ Junichi Hata, ${ }^{2,8}$ Tsunehiko Konomi, ${ }^{1,2,6}$ \\ Toshihiro Nagai, ${ }^{5}$ Shinsuke Shibata, ${ }^{2}$ Shinjiro Kaneko, ${ }^{6}$ Akio Iwanami, ${ }^{1}$ Suketaka Momoshima, ${ }^{4}$ Shinichi Takahashi, ${ }^{3}$ \\ Masahiro Jinzaki, ${ }^{4}$ @Norihiro Suzuki, ${ }^{3}$ Yoshiaki Toyama, ${ }^{1}{ }^{\circ}$ Masaya Nakamura, ${ }^{1}$ and Hideyuki Okano ${ }^{2,8}$ \\ Departments of ${ }^{1}$ Orthopedic Surgery, ${ }^{2}$ Physiology, ${ }^{3}$ Neurology, and ${ }^{4}$ Radiology, and ${ }^{5}$ Electron Microscopic Laboratory, Keio University School of Medicine, \\ Tokyo 160-8582, Japan, ${ }^{6}$ Department of Orthopedic Surgery, National Hospital Organization Murayama Medical Center, Tokyo 208-0011, Japan, ${ }^{7}$ Central \\ Institute for Experimental Animals, Kanagawa 210-0821, Japan, and ${ }^{8}$ Laboratory for Marmoset Neural Architecture, RIKEN Brain Science Institute, \\ Saitama 351-0198, Japan
}

White matter abnormalities in the CNS have been reported recently in various neurological and psychiatric disorders. Quantitation of nonGaussianity for water diffusion by $q$-space diffusional MRI (QSI) renders biological diffusion barriers such as myelin sheaths; however, the time-consuming nature of this method hinders its clinical application. In the current study, we aimed to refine QSI protocols to enable their clinical application and to visualize myelin signals in a clinical setting. For this purpose, animal studies were first performed to optimize the acquisition protocol of a non-Gaussian QSI metric. The heat map of standardized kurtosis values derived from optimal QSI (myelin map) was then created. Histological validation of the myelin map was performed in myelin-deficient mice and in a nonhuman primate by monitoring its variation during demyelination and remyelination after chemical spinal cord injury. The results demonstrated that it was sensitive enough to depict dysmyelination, demyelination, and remyelination in animal models. Finally, its utility in clinical practice was assessed by a pilot clinical study in a selected group of patients with multiple sclerosis (MS). The human myelin map could be obtained within 10 min with a $3 \mathrm{~T}$ MR scanner. Use of the myelin map was practical for visualizing white matter and it sensitively detected reappearance of myelin signals after demyelination, possibly reflecting remyelination in MS patients. Our results together suggest that the myelin map, a kurtosis-related heat map obtainable with time-saving QSI, may be a novel and clinically useful means of visualizing myelin in the human CNS.

Key words: demyelination; MRI; multiple sclerosis; myelin; remyelination

Significance Statement

Myelin abnormalities in the CNS have been gaining increasing attention in various neurological and psychiatric diseases. However, appropriate methods with which to monitor CNS myelin in daily clinical practice have been lacking. In the current study, we introduced a novel MRI modality that produces the "myelin map." The myelin map accurately depicted myelin status in mice and nonhuman primates and in a pilot clinical study of multiple sclerosis patients, suggesting that it is useful in detecting possibly remyelinated lesions. A myelin map of the human brain could be obtained in $<10$ min using a $3 \mathrm{~T}$ scanner and it therefore promises to be a powerful tool for researchers and clinicians examining myelin-related diseases.

\section{Introduction}

Myelin in the CNS not only enables saltatory conduction, but also plays an essential role in supporting neural survival (Lee et al.,

Received May 6, 2015; revised Dec. 22, 2015; accepted Jan. 29, 2016.

Author contributions: M.N. and H.O. designed research; K.F., K.H., J.N., O.T., J.H., T.K., T.N., S.S., S.K., A.I., S.M., S.T., M.J., N.S., Y.T., M.N., and H.O. performed research; K.F., K.H., J.N., O.T., J.H., T.K., T.N., S.S., S.K., A.I., S.M., S.T., M.J., N.S., Y.T., M.N. and H.O. analyzed data; K.F., K.H., J.N., O.T., J.H., T.K., T.N., S.S., S.K., A.I., S.M., S.T., M.J., N.S., Y.T., M.N. and H.O. wrote the paper.

This study was supported by the Ministry of Education, Culture, Sports, Science and Technology (MEXT) of Japan and the Japan Agency for Medical Research and Development (AMED), including the Strategic Research Program for Brain Sciences, Brain Mapping by Integrated Neurotechnologies for Disease Studies (Brain/ MINDS; H.O., J.H., and S.S.), the Research (enter Network for Realization of Regenerative Medicine (H.O.), and
2012). Myelin failure preceding neurodegeneration was reported in amyotrophic lateral sclerosis (Kang et al., 2013). Collectively, myelin pathology is attracting significant attention, not only in the context of demyelinating diseases such as multiple sclerosis (MS), but also in the setting of various neurological diseases.

There have been attempts to detect myelin-specific signals by nonconventional magnetic resonance methods such as

a Grant-in-Aid for Scientific Research (J.N.); the CREST program from the Japan Science and Technology Agency (M.N.); and by the General Insurance Association of Japan (Y.T. and M.N.). We thank Hiroko Baba for providing the shiverer mutant mice, Kazuhiro Ikenaka and Kenji Tanaka for providing the jimpy mutant mice, Hiroyuki Kato and Seiji Okada for editing and proofreading the manuscript, and Kaori Yasutake for her expertise in animal experiments. 


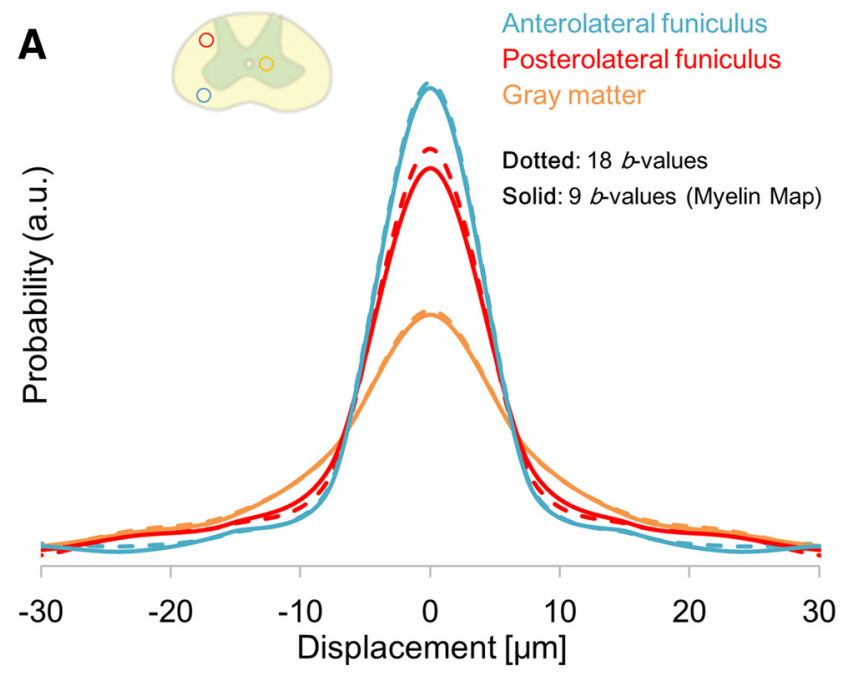

B

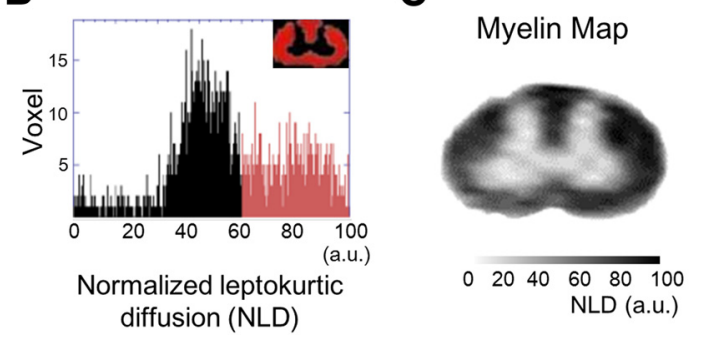

Figure 1. Development of the myelin map. The PDF of water diffusion acquired from the cervical spinal cord in adult common marmosets reveals that the diffusion distribution of white matter (anterolateral and posterolateral funiculi) is more leptokurtic than that of gray matter $(A)$. Note that PDF curves obtained with a time-saving protocol ( $9 b$-values; hereafter used for the myelin map; solid lines) had sufficient accuracy to differentiate gray and white matter when compared with the time-consuming full-scale protocol (18 $b$-values; dotted lines). The histogram of NLD calculated from the PDF is shown in $\boldsymbol{B}$. The NLD heat map and the myelin map created from the PDF are shown in $\boldsymbol{C}$. a.u., Arbitrary units.

magnetization transfer ratio analysis (Gass et al., 1994) and T2 relaxation study (Laule et al., 2004) or positron emission tomography (Stankoff et al., 2011), although the application of these methods in daily clinical practice has been hindered by their time-consuming nature (e.g., hours of acquisition time), relatively poor resolution, or the need to use radioactive tracers. Conversely, visualization of myelin by conventional MRI has technical limitations. For example, although T2 hyperintensity is often interpreted as a hallmark of demyelination, a postmortem MRI study showed that remyelinated MS lesions were also T2 hyperintense (Barkhof et al., 2003) and T2 lesion loads did not necessary correlate with physical disability measures in MS (Barkhof, 1999).

Myelin is a lipid-enriched structure that hinders the free diffusion of water molecules. Theoretically, diffusion is more restricted in myelinated than nonmyelinated areas. The apparent diffusion coefficient calculated by diffusion-weighted

J.N. received honoraria from Novartis Pharma, Bayer Pharmaceutical Japan, Mitsubishi Tanabe Pharma, and Takeda Pharmaceutical and serves as a paid scientific advisor to Novartis Pharma, Bayer Pharmaceutical Japan, Takeda Pharmaceutical, and Biogen Japan. H.O. serves as a paid scientific advisor to SanBio Co. Ltd. The remaining authors declare no competing financial interests.

*K.F., K.H, and J.N. made equal contributions to this work.

Correspondence should be addressed to either Dr. Masaya Nakamura (Department of Orthopedic Surgery) or Dr. Hideyuki Okano (Department of Physiology), Keio University School of Medicine, 35 Shinanomachi, Shinjuku, Tokyo 160-8582, Japan. E-mail:masa@a8.keio.jp or hidokano@a2.keio.jp.

DOI:10.1523/JNEUROSCI.1770-15.2016

Copyright $\odot 2016$ the authors $\quad 0270-6474 / 16 / 362797-13 \$ 15.00 / 0$ imaging (DWI) is a straightforward parameter of diffusion restriction. Fractional anisotropy (FA), derived using diffusion tensor imaging (DTI), is a parameter describing the degree of anisotropy of diffusion restriction. These parameters have been used to estimate the presence of the biological diffusion barrier myelin within the human CNS (Pfefferbaum et al., 2000; Engelbrecht et al., 2002). However, they are based on an assumption that the probability density function (PDF) of water diffusion is Gaussian in its shape. In reality, water diffusion is not only restricted by myelin, but also by cell membranes or by axonal sheaths, thereby producing nonGaussianity in the PDF.

$q$-Space diffusion MRI (QSI) enables the calculation of the PDF in a non-Gaussian fashion (King et al., 1994). The key principle of QSI is that the PDF is derived from a Fourier transformation of a diffusion-related signal decay curve with respect to multiple $b$-values. The PDF obtained by QSI provides an ideal metric with which to more accurately estimate the presence of myelin. For example, the difference in the PDFs of gray and white matter is well correlated with myelination during postnatal CNS development (Assaf et al., 2000). This difference is lost when demyelination occurs and is then regained when remyelination takes place after traumatic CNS injury, with good correlation with histological myelin status (Nossin-Manor et al., 2002). Despite the impressive ability of QSI to visualize CNS myelin, its time-consuming nature hinders its clinical application. Diffusional kurtosis imaging (DKI) was developed to compensate for this limitation (Jensen et al., 2005). Kurtosis is a metric of non-Gaussianity that represents the deviation from a Gaussian curve. DKI reduces the long acquisition times involved in full-scale QSI by using a rather simplified DWI sequence with a limited number of low $b$-values ( $\sim 2000 \mathrm{~s} / \mathrm{mm}^{2}, 3$ steps $)$. The major limitation of DKI is that the estimation equation is only valid for low $b$-values $\left(\sim 3500 \mathrm{~s} / \mathrm{mm}^{2}\right)$ (Hori et al., 2012b). However, the diffusionrelated signal decay curve in white matter is multiexponential and the accuracy of the PDF depends upon data with high $b$-values $\left(\sim 10,000 \mathrm{~s} / \mathrm{mm}^{2}\right)$ (Cohen et al., 2002). As a result, diffusion resolution is sacrificed in DKI.

In the current study, we sought to combine the merits of both DKI and full-scale QSI to provide a new modality for myelin visualization. To reduce the acquisition time, we applied high $b$-values while limiting their number $\left(\sim 10,000 \mathrm{~s} / \mathrm{mm}^{2}, 9\right.$ steps $)$. The heat map of standardized kurtosis values derived from optimal QSI (i.e., myelin map) correlated with the histological myelin status in animals. The proposed QSI protocol can be performed with a conventional $3 \mathrm{~T}$ MR scanner within $10 \mathrm{~min}$, and the myelin map seemed to reflect the myelin status in MS patients accurately. We propose the use of this new modality in clinical settings for the effective determination of myelin status in various CNS diseases.

\section{Materials and Methods}

Ethics. All animal interventions and care were performed at the Central Institute of Experimental Animals (CIEA), Kawasaki, Japan. All study protocols were approved by the institutional Animal Care and Use committee at CIEA and conducted in accordance with the United States Public Health Service's Policy on Human Care and the Use of Laboratory Animals and Guidelines and Policies for Animal Surgery provided by the Animal Study Committee of CIEA. The clinical study was performed at Keio University Hospital, Tokyo, Japan, with adequate written informed consent and was approved in advance by the Institutional Review Board of Keio University School of Medicine (approval number: 20110364). 

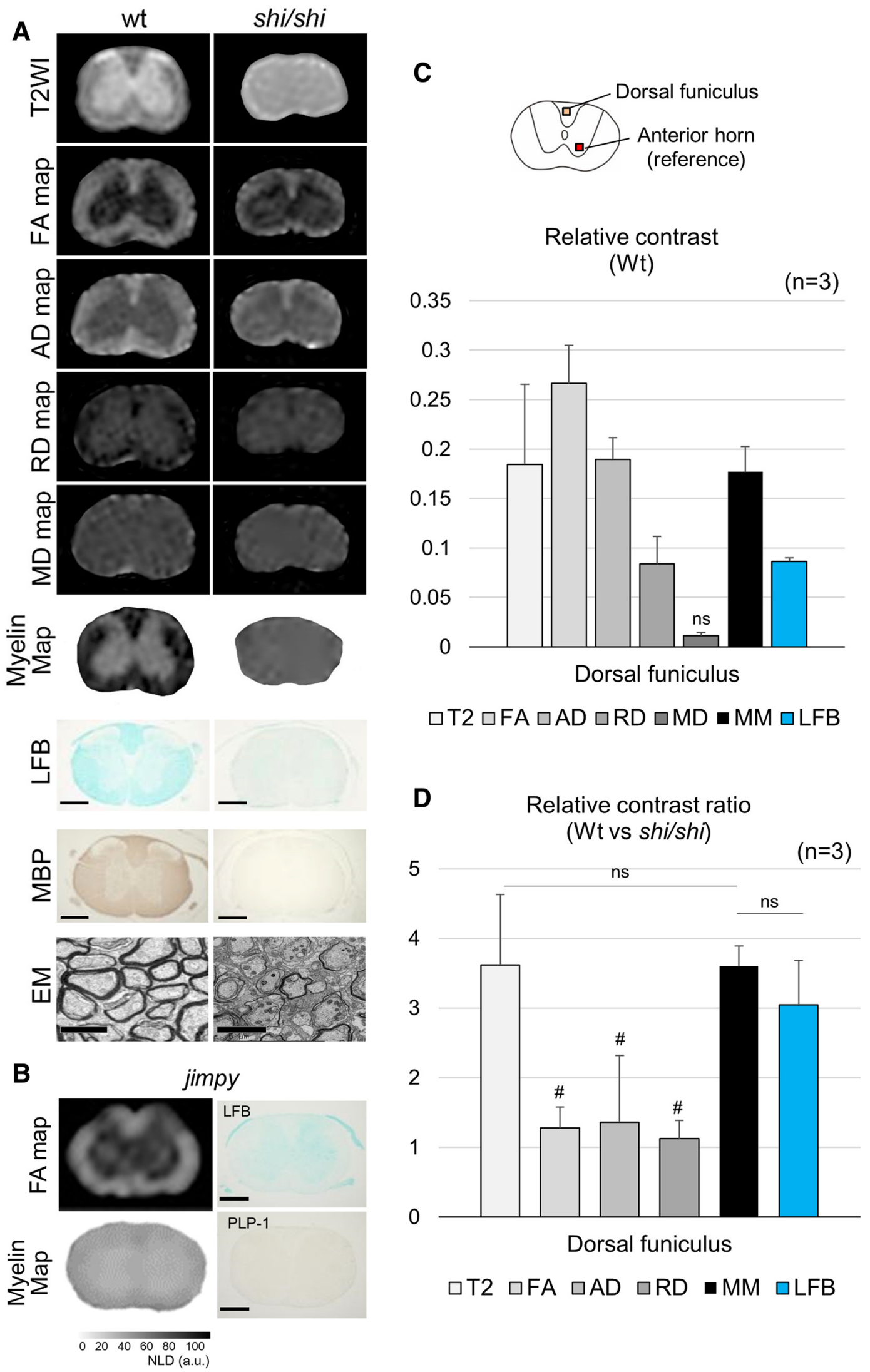

Figure 2. Spinal cord myelin map of myelin-deficient mutant mice. T2WI, FA map, various DTI maps (i.e., AD map, RD map, and MD map), and the myelin map, as well as LFB staining, immunohistochemical staining against MBP, and electron microscopic (EM) analysis of postmortem spinal cords of wild-type (wt) and shiverer (shi/shi) mutant mice are shown in $A$. For quantitative analysis, the sensitivity of various modalities to differentiate white matter from gray matter was discerned using relative contrast values, calculated by first determining the absolute difference in 8-bit grayscale values (GV; $0-255$ ) between ROls (dorsal funiculus; orange box) and a reference (anterior horn; red box) and then dividing this value by 255 . Relative contrast values exceeding 0.05 were considered to be clinically significant. All of the MRI modalities, with the exception of the MD map, were considered to be significant ( $\boldsymbol{C}$ ). To elucidate the myelin (Figure legend continues.) 


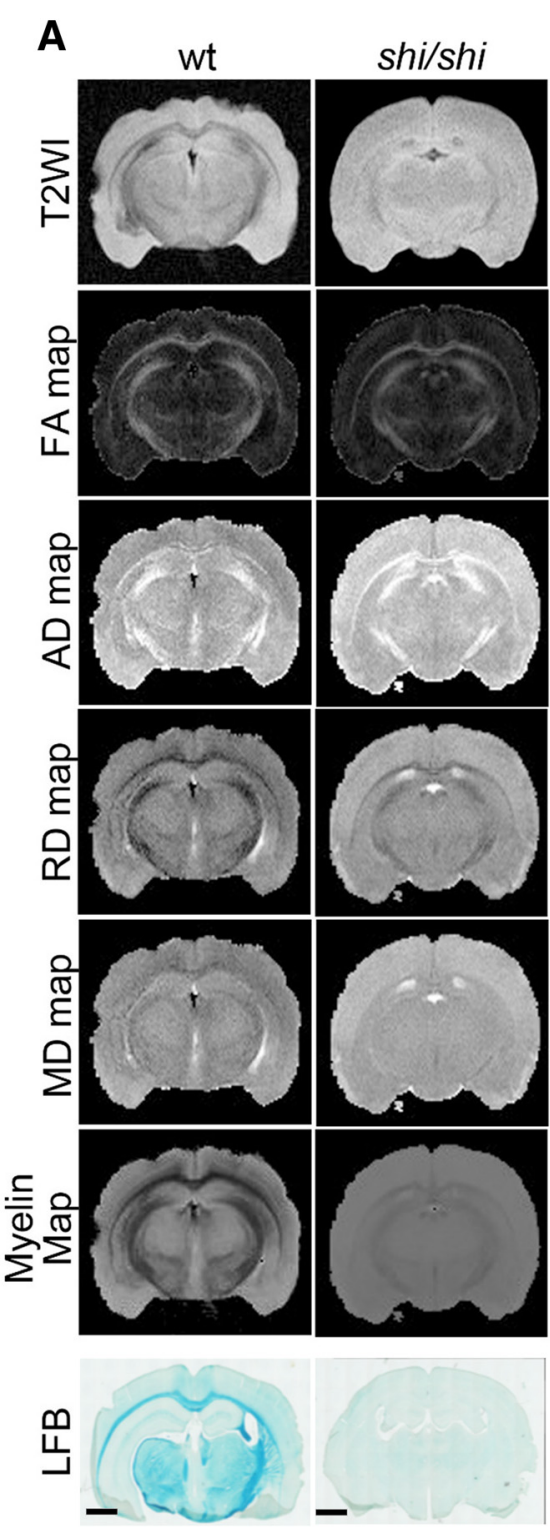

B

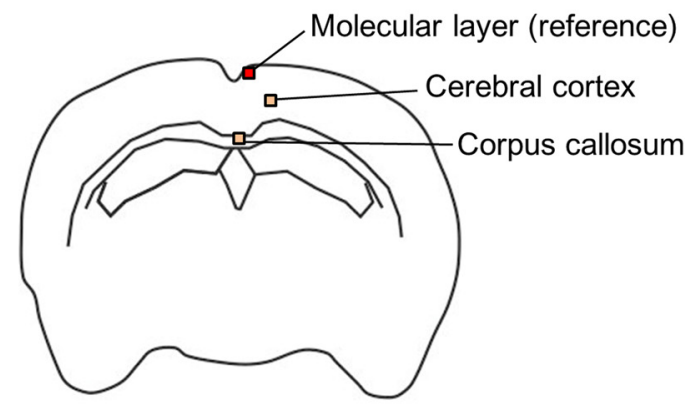

Relative contrast $(\mathrm{Wt})$

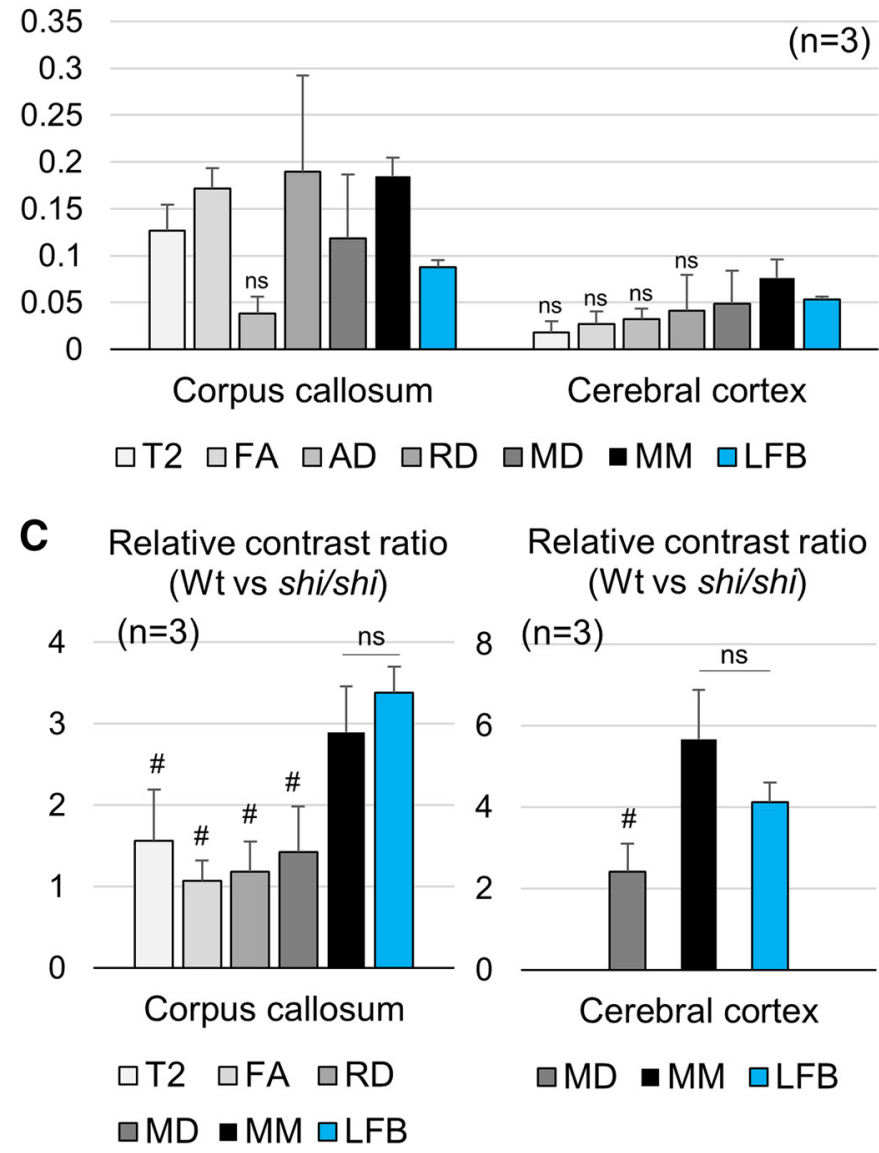

Figure 3. Brain myelin map of myelin-deficient Shiverer mutant mice. T2WI, FA map, various DTI maps (i.e., AD map, RD map, and MD map), the myelin map, and histological LFB staining of postmortem wild-type (wt) and shiverer (shi/shi) mouse brains are shown $(\boldsymbol{A})$. For quantitative analysis, relative contrast values were calculated similarly to the spinal cord analysis (Fig. 2). Myelinated fibers in the corpus callosum and cerebral cortex were selected as Rols (orange boxes) and the molecular layer of cerebral cortex, in which few myelinated fibers exist, was used as a reference (red box). Relative contrast values exceeding 0.05 were considered to be clinically significant. All of the MRI modalities, with the exception of the AD map, were considered to be significant in the corpus callosum, whereas only the myelin map and MD map were significant in the cerebral cortex (B). Relative contrast ratios were calculated similarly to the spinal cord analysis (Fig. 2). Results indicated that the myelin map was the most myelin-specific modality, with a ratio that was fairly comparable to that of histological LFB staining (C). Scale bar, $1 \mathrm{~mm}$. \#p $<0.05$, Mann-Whitney $U$ test. Error bars indicate SEM.

$\leftarrow$

(Figure legend continued.) specificity of observed contrast, relative contrast values were compared between wt and shi/shi mice. Results indicated that the myelin map had superior myelin specificity relative to the FA, AD, and RD maps, whereas T2WI and LFB had similar specificity compared with the myelin map (D). Comparison of the FA map, the myelin map, LFB staining, and immunostaining against PLP-1 in jimpy mutant mice also confirmed the high accuracy of the myelin map in detecting myelin-related signals $(\boldsymbol{B})$. Scale bars: LFB, MBP, and PLP-1, $500 \mu \mathrm{m}$; EM, $5 \mu \mathrm{m}$. a.u., Arbitrary units. \#p $<0.05$, Mann-Whitney $U$ test. Error bars indicate SEM.
Animals. The following animals were used in this study: common marmosets (Callithrix jacchus; CLEA Japan), shiverer and jimpy mutant mice (The Jackson Laboratory), and their wild-type littermates.

Chemically induced demyelination in common marmosets. A total of six adult female common marmosets $(270-360 \mathrm{~g})$ were used. All surgeries and MRI acquisitions were performed under general anesthesia induced by an intramuscular injection of ketamine ( $50 \mathrm{mg} / \mathrm{kg}$; Sankyo) and xylazine $(5 \mathrm{mg} / \mathrm{kg}$; Bayer) and maintained using isoflurane (Foren; Abbott Japan). The heart rate, arterial oxygen saturation, and rectal temperature of each animal were monitored continuously during the surgical procedures. For the induction of focal demyelination, the dura mater and pia at 
the C5 level were incised with a sharp needle and $3 \mu \mathrm{l}$ of $1 \%$ lysophatidylcholine (LPC; Sigma-Aldrich) was injected at a depth of 1.0 $\mathrm{mm}$ into the dorsal funiculus of the spinal cord using a glass micropipette attached to a Hamilton syringe. The injection was performed slowly, over a $10 \mathrm{~min}$ period and the micropipette was held in place for an additional $10 \mathrm{~min}$ to prevent backflow.

Myelin map. The non-Gaussian PDF of water diffusion was obtained by performing a Fourier transformation of the data gained from a pulse-field gradient spin-echo (PGSE) sequence based on the Stejskal-Tanner diffusion preparation (Stejskal and Tanner, 1965), performed along 12 motion-probing gradient (MPG) axes with 9 or $18 b$-value steps $(0-$ $10,000 \mathrm{~s} / \mathrm{mm}^{2}$ ). An in-house program (developed in IDL version 8.0; Exelis Visual Information Solutions) was used to perform further analyses. Kurtosis values were calculated with the following formula:

$$
K=\frac{1}{N} \sum\left(\frac{x_{i}-\bar{x}}{S D}\right)^{4}-3
$$

Where $N$ is the number of data points (i.e., the number of $b$-value steps used), $x$ is the probability value (arbitrary units) obtained from the $\mathrm{PDF}$, and SD is the SD of the $x$ values. To standardize the kurtosis value, we calculated the normalized leptokurtic diffusion (NLD) with the following formula:

$$
\mathrm{NLD}=\frac{\left(K_{\max }-K\right)}{\left(K_{\max }-K_{\min }\right)} * 100
$$

Where $K_{\max }$ and $K_{\min }$ are the maximum and minimum, respectively, kurtosis values obtained in multiple control studies (note that $K_{\max }$ and $K_{\min }$ are species dependent) and $K$ is the mean kurtosis value across all directions obtained in the individual experiment. The myelin map was defined as the NLD heat map created by linearly coloring NLD values (i.e., grayscale and color-coded images were made with an NLD value of 0 corresponding to white and 100 corresponding to either black or blue, respectively).

Animal MRI parameters. An MR scanner equipped with a $7 \mathrm{~T}$ magnet with a high gradient strength (700 $\mathrm{mT} / \mathrm{m})$ (Biospec 70/16; Bruker) was used. A $60 \mathrm{~mm}$ inner diameter (ID) and $22 \mathrm{~mm}$ ID saddle coil, tuned to 300.51 $\mathrm{MHz}$ for proton resonance, was used for in vivo and ex vivo measurements, respectively. For the ex vivo measurement, excised mouse brain and spinal cord were inserted into a $20 \mathrm{~mm}$ ID acrylic tube containing electronic liquid (Fluorinert FC-72; 3M) and were immediately subjected to imaging. The PGSE sequence used the following parameters: repetition time $(\mathrm{TR})=$ $3500 \mathrm{~ms}$, echo time $(\mathrm{TE})=37.8 \mathrm{~ms}$, matrix $=$ $256 \times 192$, field of view $(\mathrm{FOV})=40 \times 40$ $\mathrm{mm}^{2}$, and slice thickness $=1.5 \mathrm{~mm}$ for in vivo measurements, and $\mathrm{TR}=3500 \mathrm{~ms}$, $\mathrm{TE}=56$ $\mathrm{ms}$, matrix $=128 \times 32, \mathrm{FOV}=19.8 \times 5 \mathrm{~mm}^{2}$, and slice thickness $=0.48 \mathrm{~mm}$ for ex vivo measurements. FA maps were acquired with a PGSE sequence based on the Stejskal-Tanner
A

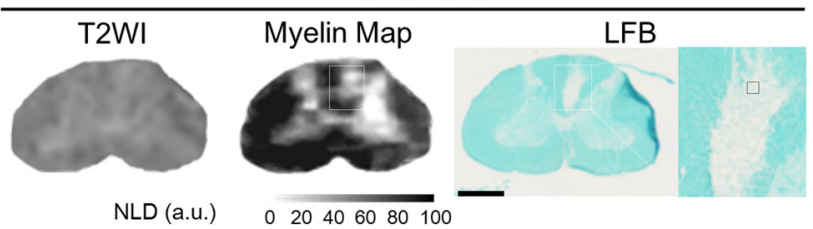

Semi-thin

EM

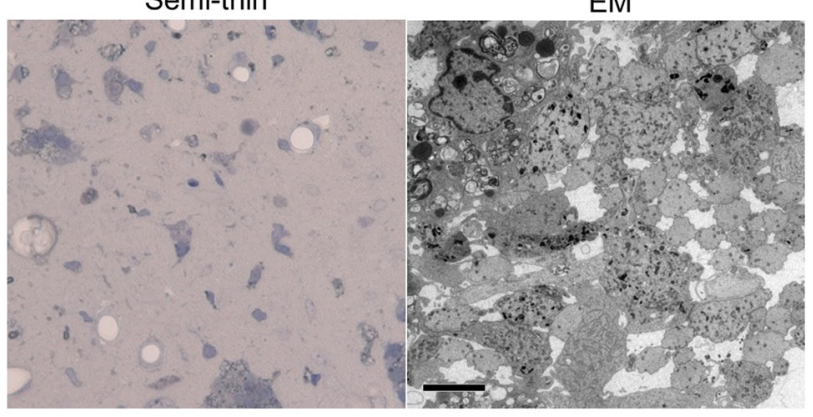

B

PI 6w
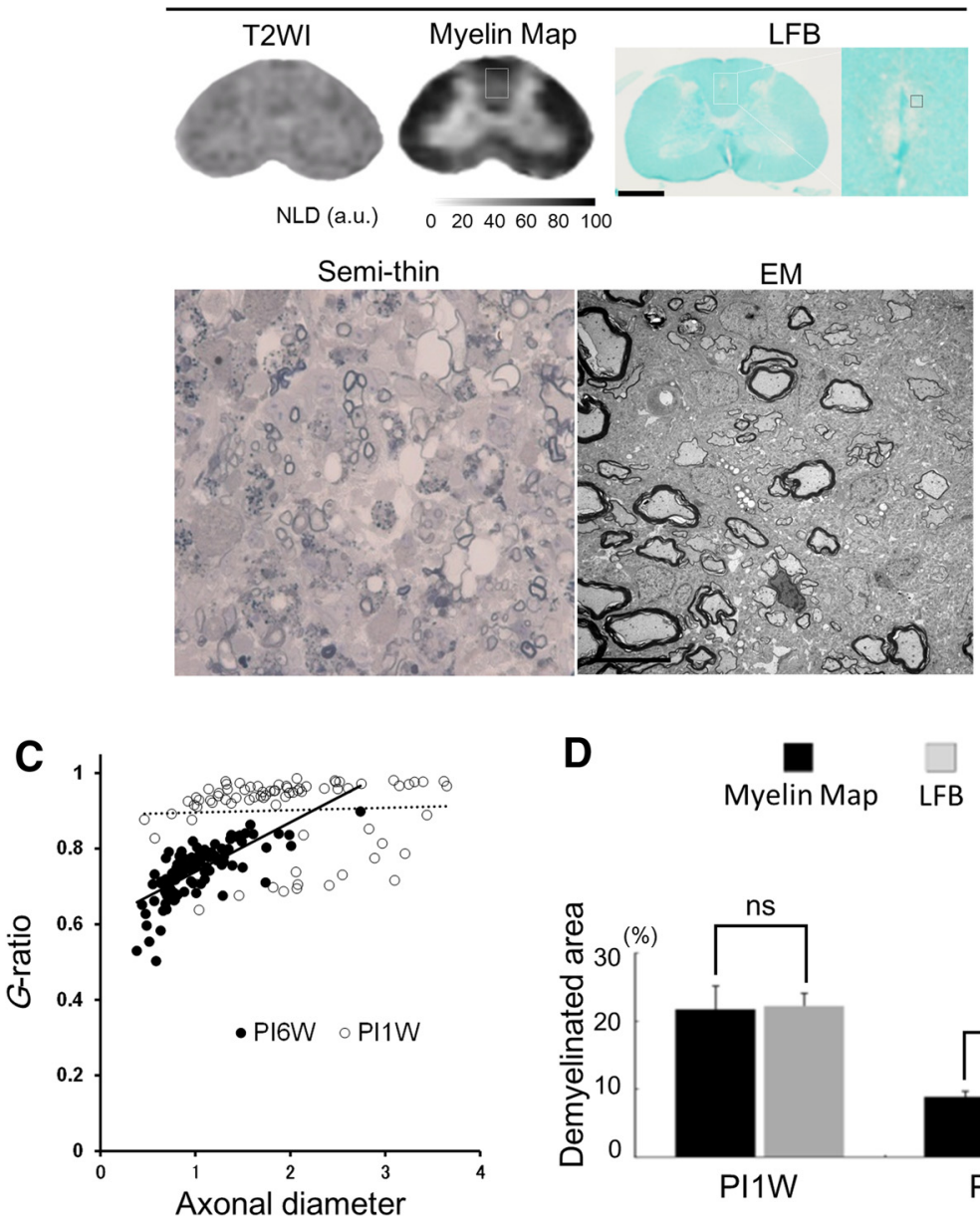

D

Myelin Map LFB

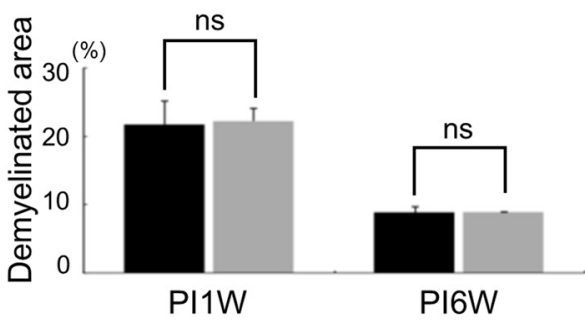

Figure 4. Myelin map of common marmosets with chemically induced focal demyelination. Axial images of spinal cord obtained 1 week after chemically induced demyelination, showing T2WI and myelin map results in a live animal, and postmortem LFB, semithin sections, and electron microscopic (EM) analysis $(\boldsymbol{A})$. The same set of axial images was obtained 6 weeks after demyelination $(\boldsymbol{B})$. The $g$-ratio, calculated by dividing axonal diameter by myelinated fiber diameter (e.g., the $g$-ratio for a completely demyelinated axon is 1$)$, shows decreased values at 6 weeks after injury ( $0.74 \pm 0.070$; mean \pm SE) compared with 1 week postinjury ( $0.90 \pm 0.095)$, suggestive of remyelination (C). Quantitative comparative analysis of demyelinated areas in the dorsal funiculus using a myelin map in a live animal and postmortem LFB ( $n=4$ each for 1 and 6 weeks after injury) suggests the high accuracy of the myelin map in detecting demyelinated areas (D). Note that T2WI failed to detect demyelinating lesions clearly in this model. a.u., Arbitrary units. Scale bars: LFB, $1 \mu \mathrm{m}$; EM, $10 \mu \mathrm{m}$. Error bars indicate SEM. 


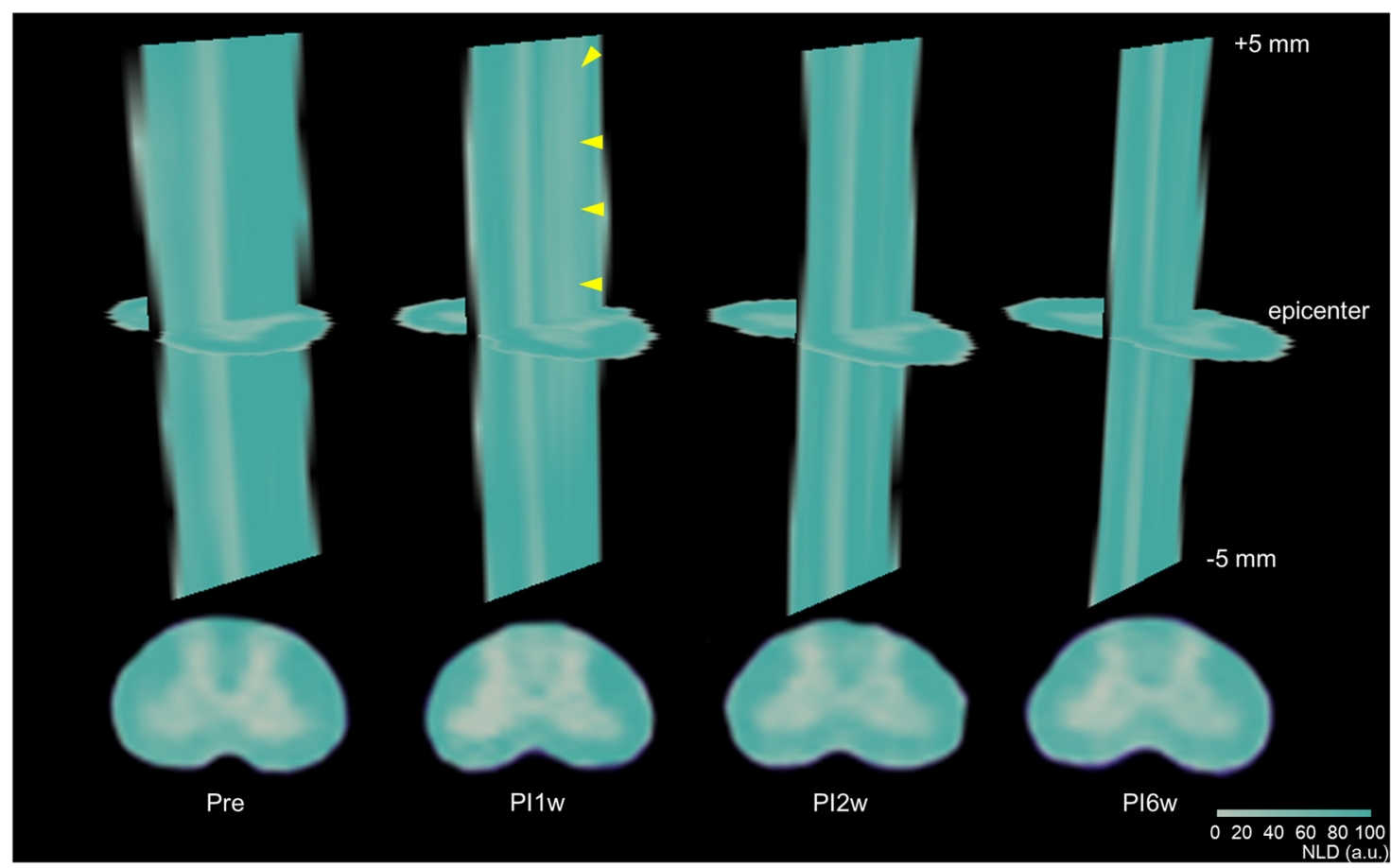

Figure 5. Remyelination activity detected in chronological myelin maps of common marmosets after chemically induced focal demyelination. Chronological, 3D, color-coded myelin maps sequentially obtained from the same animal as in Figure 4 reveal signal loss due to chemical demyelination in the dorsal funiculus at 1 week (arrowheads), as well as signal recovery at 6 weeks. a.u., Arbitrary units.

diffusion preparation (Stejskal and Tanner, 1965) performed along 12 MPG axes with a $b$-value of 0 or $1000 \mathrm{~s} / \mathrm{mm}^{2}$ with TR $=3500 \mathrm{~ms}$, TE $=$ $26.8 \mathrm{~ms}$, matrix $=128 \times 32, \mathrm{FOV}=19.8 \times 5 \mathrm{~mm}^{2}$, and slice thickness $=$ $0.48 \mathrm{~mm}$. FA maps were computed using the Diffusion Toolkit (Massachusetts General Hospital). For DTI, an eigenvector associated with the largest eigenvalue $(\lambda 1)$ was assumed to represent the local fiber direction (e.g., $\lambda 1$ is parallel to the long axis of the spinal cord, whereas $\lambda 2$ and $\lambda 3$ are perpendicular). DTI results [axial diffusivity (AD) map, radial diffusivity $(\mathrm{RD})$ map, and mean diffusivity $(\mathrm{MD})$ map] were reconstructed on the basis of the data as follows: $\mathrm{AD}=\lambda 1, \mathrm{RD}=(\lambda 2+\lambda 3) / 2$, and $\mathrm{MD}=$ $(\lambda 1+\lambda 2+\lambda 3) / 3$.

Human MRI parameters. An MR scanner equipped with a $3 \mathrm{~T}$ magnet and 32-channel head coil (Discovery MR750; GE Healthcare) was used. The PGSE sequence used TR $=5000 \mathrm{~ms}$, TE $=124 \mathrm{~ms}$, matrix $=128 \times$ $128, \mathrm{FOV}=220 \times 220 \mathrm{~mm}^{2}$, and slice thickness $=5 \mathrm{~mm}$ along $12 \mathrm{MPG}$ axes, with $b$-values ranging from 0 to $10,000 \mathrm{~s} / \mathrm{mm}^{2}$ in 9 steps. The total acquisition time to obtain the dataset for the myelin map was $\sim 9$ min. The dataset for FA maps and other DTI maps was acquired with the same PGSE sequence performed along 12 MPG axes with a $b$-value of 0 or 1066 $\mathrm{s} / \mathrm{mm}^{2}$. FA maps were computed using the Diffusion Toolkit and DTI results were reconstructed similarly to animal MRI. Additional T2weighted images $(\mathrm{T} 2 \mathrm{WI}$; TR $=5000 \mathrm{~ms}, \mathrm{TE}=93 \mathrm{~ms}$, matrix $=384 \times$ 288, slice thickness $=5 \mathrm{~mm})$ and T1-weighted images $(\mathrm{T} 1 \mathrm{WI} ; \mathrm{TR}=580$ $\mathrm{ms}, \mathrm{TE}=10 \mathrm{~ms}$, matrix $=288 \times 192)$ were acquired for comparison. Gadolinium-based contrast agents (Omniscan; Daiichi-Sankyo) were used when necessary and the injection was always made at the end of the scan to avoid a possible influence of the agent on myelin map signals.

Quantitative comparison of MRI modalities. For quantitative comparison of MRI modalities, the relative contrast value was calculated with the following formula:

$$
\text { relative contrast }=\frac{\left|G V_{\mathrm{ROI}}-G V_{\mathrm{REF}}\right|}{255}
$$

Where $G V_{\mathrm{ROI}}$ and $G V_{\mathrm{REF}}$ are median gray values (0-255; an 8-bit grayscale) measured with Photoshop software (version CS5 extended; Adobe) in ROIs and reference areas (regions with few myelinated fibers; i.e., anterior horn for mouse spinal cords, molecular layer of cerebral cortex for mouse brains, and CSF in cerebral ventricles for human brains). Relative contrast was calculated for 3 consecutive slices per individual for each modality (i.e., T2WI, FA map, AD map, RD map, MD map, and the myelin map) and 3 sets of wild-type and shiverer mutant mice or 5 volunteers (young adults; 4 females and 1 male) were analyzed. Mean relative contrast exceeding 0.05 was considered to be clinically significant. For mice, relative contrast ratio was calculated to determine the myelin specificity by dividing the relative contrast value of wild-type mice by that of myelin-deficient shiverer mutant mice. For statistical analysis, Mann-Whitney $U$ tests were performed to compare relative contrast values or ratios and $p<0.05$ was considered to be statistically significant.

Histological analysis. Each animal was intracardially perfused with $4 \%$ paraformaldehyde (4\% PFA; Wako Pure Chemical Industries) and the brain and/or spinal cord was removed. The dissected specimen was postfixed for $3 \mathrm{~h}$ in $4 \%$ PFA, soaked overnight in $10 \%$ sucrose (Wako) in PBS (Wako) followed by $30 \%$ sucrose in PBS, and cut into $20-\mu \mathrm{m}$-thick sections using a cryostat (Leica). Tissue sections were stained with Luxol fast blue (LFB; Muto Pure Chemicals) to evaluate the myelinated area. Immunohistochemistry was performed with standard procedures. The antibodies used in this study were anti-myelin basic protein (MBP) chicken IgY (Aves Labs), anti-proteolipid protein (PLP) mouse IgG (Millipore), and anti-chicken IgY and anti-mouse IgG secondary antibodies (Jackson ImmunoResearch Laboratories). For the quantitative analysis of demyelinated areas, images were captured and analyzed with an optical microscope equipped with analytic software (BIOREVO BZ9000; Keyence). For the analysis, the ROI was set on the entire dorsal funiculus. For comparison, demyelinated areas were presumed to be those below $\mathrm{NLD}=50$ on the myelin map. Student's $t$ test was used for the statistical analysis. For comparison with various MRI modalities, the relative contrast value was calculated for LFB pictures linearly converted to an 8-bit grayscale using Adobe Photoshop software.

Quantification of myelination by electron microscopy. Spinal cord tissues were processed with the standard procedure for electron microscopic analysis as described previously (Shibata et al., 2015). Briefly, samples were postfixed in $2.5 \%$ glutaraldehyde (Electron Microscopy Sciences) and $0.5 \%$ osmium tetroxide (Wako), dehydrated through a 
A
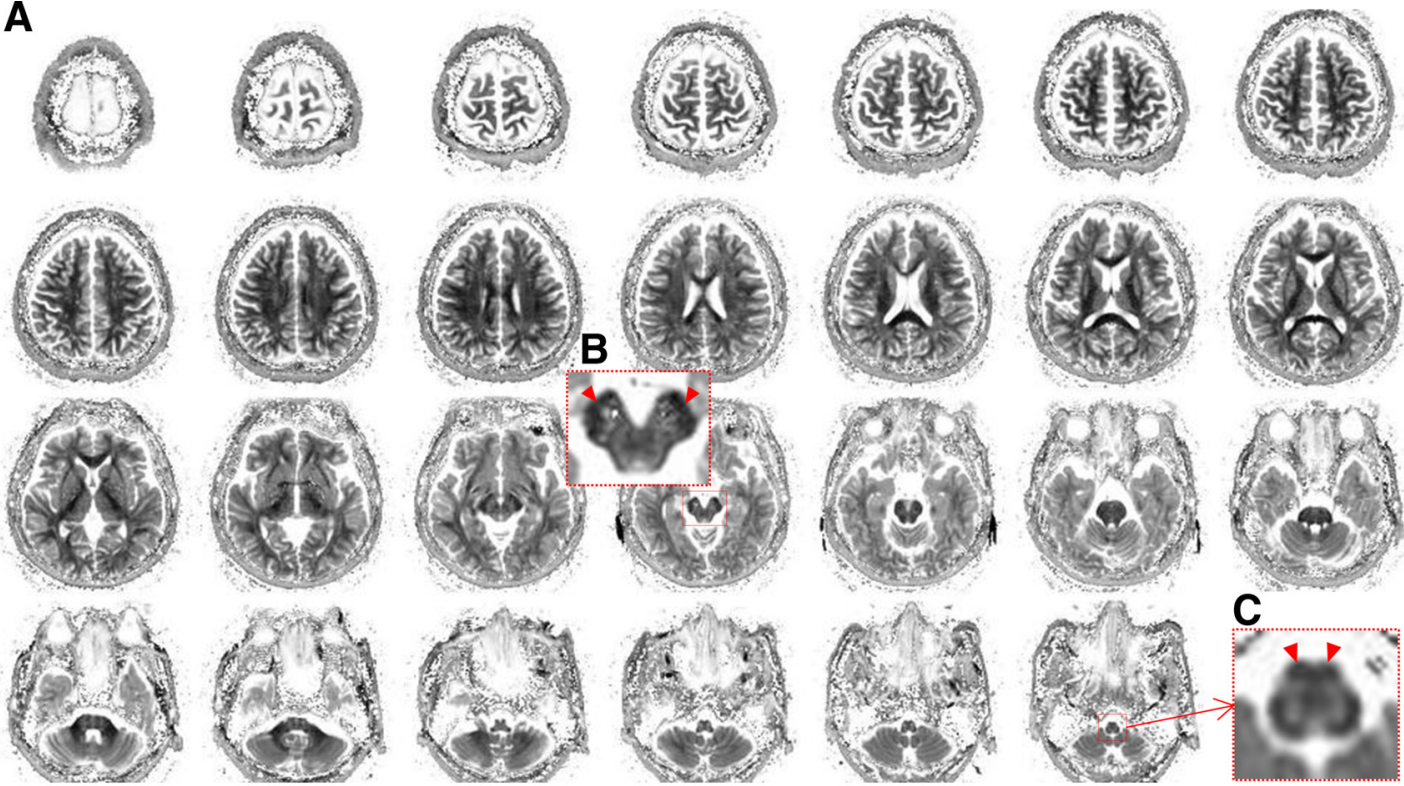

D

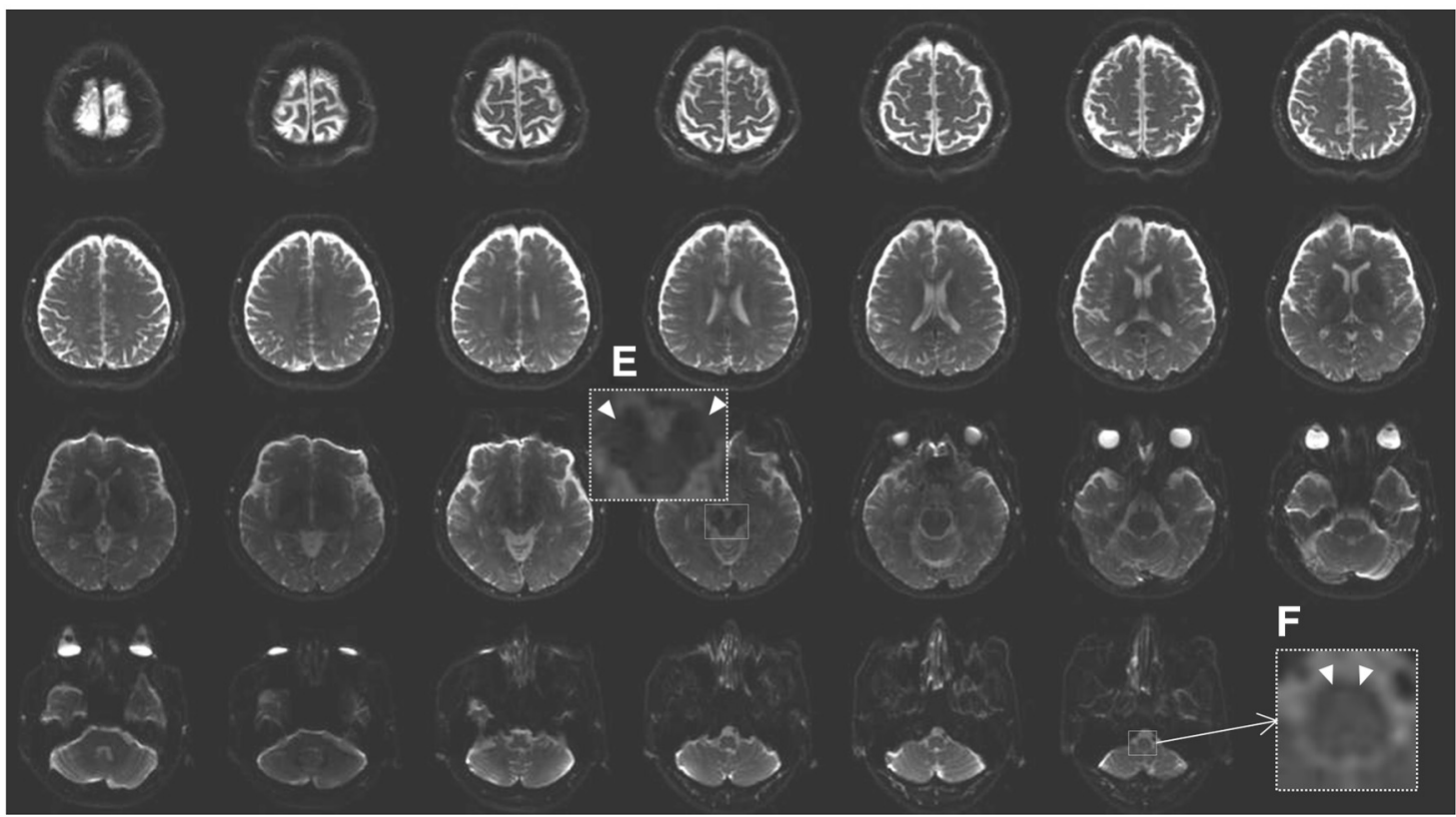

Figure 6. Myelin map of normal human brain. The whole-brain myelin map of a 40-year-old healthy male volunteer is shown $(\boldsymbol{A})$. Note that the high sensitivity of the myelin map allows detection of myelin signals in the cerebral peduncles of the midbrain and in the pyramidal tracts in the medulla oblongata ( $\boldsymbol{B}$ and $\boldsymbol{C}$, respectively). The corresponding $T 2$-weighted images are shown in $\boldsymbol{D}-\boldsymbol{F}$.

series of ethalnol solutions, and embedded in EPON (Wako). After polymerization, semithin sections (350 nm thickness) were stained with toluidine blue (Sigma-Aldrich) and images were captured with an optical microscope (BIOREVO BZ9000). Ultrathin sections were prepared, stained with uranyl acetate (Wako) and lead citrate (Sigma-Aldrich), and then observed under a transmission electron microscope (model 1230; JEOL) equipped with a digital camera (Ultrascan 4000; Gatan). The state of myelination was compared quantitatively among samples by determining $g$-ratios, which were calculated by dividing the axonal diameter by the myelinated fiber diameter, both of which were measured using 32 electron micrographs ( $2000 \times$ magnification) of randomly selected areas of all the demyelinated and remyelinated fibers as described previously (Marcus et al., 2006; Câmara et al., 2009; Liu et al., 2012). A total of 76 and 100 randomly chosen marmoset axons were analyzed at 1 week and 6 weeks after injury, respectively.

\section{Results}

QSI-based kurtosis heat map as myelin map

Diffusion-related signal decay curves are multiexponential, especially in white matter (Cohen et al., 2002), so a high $b$-value QSI, along with a series of $b$-values, is essentially required for maximum accuracy. Using a larger number of greater magnitude $b$-values permits the drawing of more precise signal decay curves from which the PDF is obtained by Fourier transformation. It is mandatory, however, to minimize the total number as well as the range of $b$-values to reduce acquisition time and thus increase clinical applicability. Conversely, according to DKI principles the kurtosis value is less influenced by the number and the range of $b$-values than by a variety of other non-Gaussianity parameters 
A
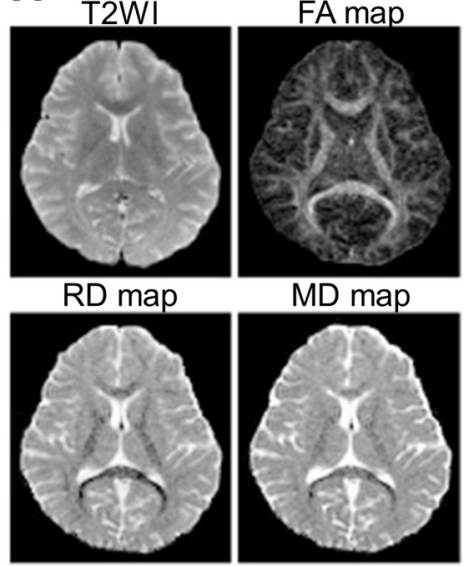

MD map
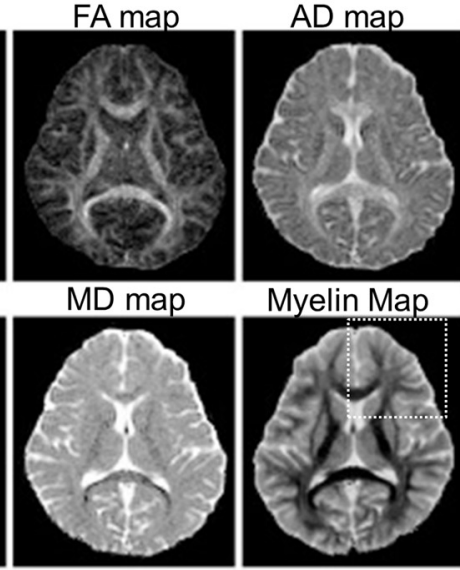

B

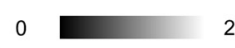

.
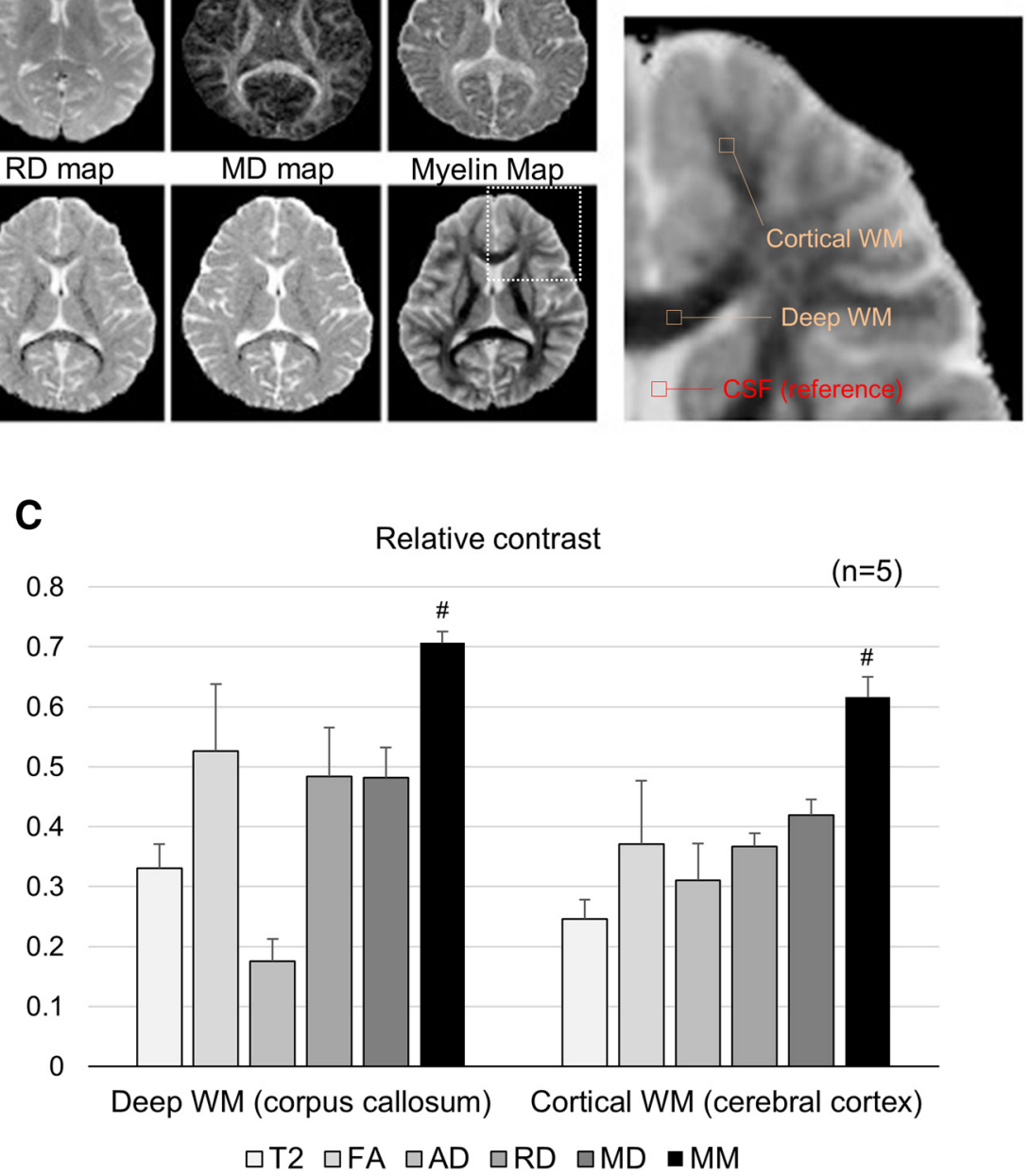

Figure 7. Comparison of myelin map, FA map, and DTI maps in normal human brain. Axial T2WI, FA map, DTI maps (i.e., AD map, RD map, and MD map), and the myelin map of a normal human brain (28-year-old healthy female volunteer) are shown (A). For quantitative analysis, the sensitivity of various modalities to differentiate myelinated areas from nonmyelinated areas was evaluated by relative contrast values, calculated by first determining the absolute difference in 8 -bit grayscale values (GV; $0-255$ ) between ROIs [orange boxes; deep white matter (WM) or cortical WM] and a reference (red box; (SF in the lateral ventricle), and then dividing this value by $255(\boldsymbol{B})$. Note that the myelin map shows superior contrast (i.e., higher relative contrast values) to the T2WI, FA map, and other DTI maps, not only in deep WM but also in cortical WM (C).\#p $<0.01$ between the myelin map compared with all other modalities by Mann-Whitney $U$ test.

(Pfefferbaum et al., 2000). The major limitation of DKI is its rather poor resolution, especially in white matter, due to the relatively low magnitude and small number of $b$-values that are used to reduce acquisition time. We therefore sought to increase clinical applicability by performing a high $b$-value QSI to obtain precise kurtosis values while reducing the number of $b$-value steps.

To optimize the QSI protocol for this purpose, we first performed a high $b$-value QSI of the spinal cord in intact common marmosets under general anesthesia with various sets of $b$-values. Previous work demonstrated that the number of $b$-value steps may be reduced to 11 (ranging from 0 to $15,000 \mathrm{~s} / \mathrm{mm}^{2}$ ) without losing the characteristic PDF curves (Hamazaki et al., 2012). To further reduce the acquisition time (to $<10 \mathrm{~min}$ in clinical applications), we were able to limit the number of $b$-value steps to 9, ranging from 0 to $10,000 \mathrm{~s} / \mathrm{mm}^{2}$, and the PDF curves obtained from this optimal QSI still differentiated white matter from gray matter with an accuracy comparable to time-consuming full-scale QSI (18 $b$-value steps; Fig. $1 A$ ). These PDF curves were more leptokurtic in white matter than in gray matter, probably due to the presence in white matter of biological diffusion barriers such as myelin.

We introduced a parameter called NLD to standardize the kurtosis values within each species. The histogram of the NLD distribution in the spinal cord of a common marmoset revealed bimodal peaks, with higher NLD values for white matter than gray matter (Fig. $1 B$ ). Finally, a heat map was created by linearly coloring the NLD values; this NLD heat map that was derived from the optimal QSI protocol was termed the myelin map. Indeed, the myelin map of a common marmoset appeared to distinguish white matter from gray matter accurately (Fig. $1 C)$.

\section{Myelin map of myelin-deficient mice}

To verify the accuracy of the myelin map rigorously, we compared its results with those of histological analysis of spinal cords obtained from postmortem myelindeficient shiverer mutant mice (defective in MBP; Kimura et al., 1985; Okano et al., 1987) and their wild-type littermates. Myelin staining with LFB, immunostaining for MBP, and EM analysis revealed a nearcomplete absence of myelin in the spinal cords of shiverer mice (Fig. 2A). To compare the ability of various MRI modalities to distinguish white matter from gray matter, the relative contrast of white matter (dorsal funiculus) was calculated by using gray matter (anterior horn) as a reference in wild-type mice (Fig. 2C). Conventional imaging methods, including T2WI, FA map, and various DTI maps, as well as the myelin map, had clinically significant relative contrast $(>0.05)$, with the exception of the MD map (Fig. $2 A, C$ ). To further verify the myelin specificity of observed contrast values, the relative contrast ratio was calculated by comparing contrast values of wild-type and myelindeficient shiverer mice (Fig. 2D). Results showed that the myelin map had higher myelin specificity than the FA, AD, and RD maps, whereas T2WI and LFB staining had similar myelin specificity as the myelin map. These results suggest that the myelin map and T2WI are the two most suitable MRI modalities for detecting myelin signals in dysmyelinated spinal cord. The same holds true for another myelin-deficient mutant mouse strain, jimpy (defective in proteolipid protein-1; Nave et al., 1987; Fig. $2 B$ and data not shown).

We also verified the utility of myelin maps of postmortem brains of wild-type and shiverer mutant mice. Considering the anisotropic nature of neural tracts within the brain, we applied MPG along 12 different axes. The myelin map, T2WI, FA map, and various DTI maps, with the exception of the AD map, had 
Table 1. MS patients included in the study

\begin{tabular}{|c|c|c|c|c|c|c|c|c|}
\hline Patient & Age/sex & MS type & Duration & EDSS & Symptoms & $\begin{array}{l}\text { Disease-modifying } \\
\text { therapy }\end{array}$ & $\begin{array}{l}\text { Positive signals on } \\
\text { myelin map }\end{array}$ & Figure(s) \\
\hline 1 & $43 / M$ & RRMS in remission & $11 y$ & 2.0 & $\begin{array}{l}\text { Full ambulatory function with dysarthria, } \\
\text { spasticity in lower extremities, paresthesia } \\
\text { in right arm, and mild truncal ataxia }\end{array}$ & Interferon $\beta$ & Yes & $8 A, 8 B, 9 A, 9 B$ \\
\hline 2 & $40 / M$ & PPMS & $5 y$ & 3.0 & $\begin{array}{l}\text { Full ambulatory function with dysarthria, } \\
\text { mild hemiparesis, and mild ataxia }\end{array}$ & Trimonthly IVMP & Yes & $8 C, 8 D, 9 C$ \\
\hline 3 & $35 / F$ & RRMS in relapse & $1 y$ & $2.0-0$ & $\begin{array}{l}\text { Full ambulatory function with weakness and } \\
\text { tactile hypoesthesia in left forearm, all } \\
\text { of which resolved after IVMP therapy }\end{array}$ & $\begin{array}{l}\text { IVMP for acute } \\
\text { relapse only }\end{array}$ & $\begin{array}{l}\text { Yes; correlated with } \\
\text { clinical recovery }\end{array}$ & 10 \\
\hline
\end{tabular}

clinically significant relative contrast values $(>0.05)$ in the corpus callosum, whereas only the myelin map and MD map were sensitive enough to depict myelinated fibers in the cerebral cortex (Fig. $3 A, B)$. Calculation of relative contrast ratios revealed that the myelin map was the most myelin-specific MRI modality in both corpus callosum and cerebral cortex, with a specificity similar to LFB (Fig. 3C). The myelin map was the most useful MRI modality to detect myelin signals in both spinal cord and brain, with a myelin specificity that was fairly comparable to that of histological LFB staining.

\section{Myelin map for monitoring myelin status in nonhuman primates}

We next investigated whether the myelin map could be used to monitor demyelination and remyelination in a live nonhuman primate (common marmoset). For this purpose, LPC was injected into the posterior funiculus of the each animal's spinal cord. LPC induces focal and transient chemical demyelination and subsequent remyelination (Hall, 1972; Watanabe et al., 2002). One week after the focal injection of LPC, the myelin map in a live common marmoset depicted a demyelinated area in the posterior funiculus. Histological analysis of the postmortem animal suggested that the myelin map had high accuracy in detecting demyelination because LFB staining confirmed a demyelinated lesion with an equivalent location and size (Fig. 4A). Six weeks after the injection of LPC, the myelin map in a live animal revealed signal recovery in the injected area.

Histological analysis of the postmortem animal confirmed the reappearance of LFB reactivity in the lesion, suggestive of remyelination, and this was further confirmed by semithin sections and EM analysis (Fig. $4 B$ ) or by $g$-ratio analysis (Fig. $4 C$ ). Comparative quantitative analysis of demyelinated areas in the posterior funiculus confirmed that the myelin map of a live animal accurately reflected the results of postmortem histological LFB analysis (Fig. 4D). We also monitored the myelin map of an individual animal with demyelination continually; sequential myelin maps were sensitive enough to visualize demyelination and remyelination (Fig. 5). T2WI failed to demarcate lesions in this demyelinating model clearly (Fig. $4 A, B$ ) despite the
$\mathrm{T} 2 \mathrm{WI}$
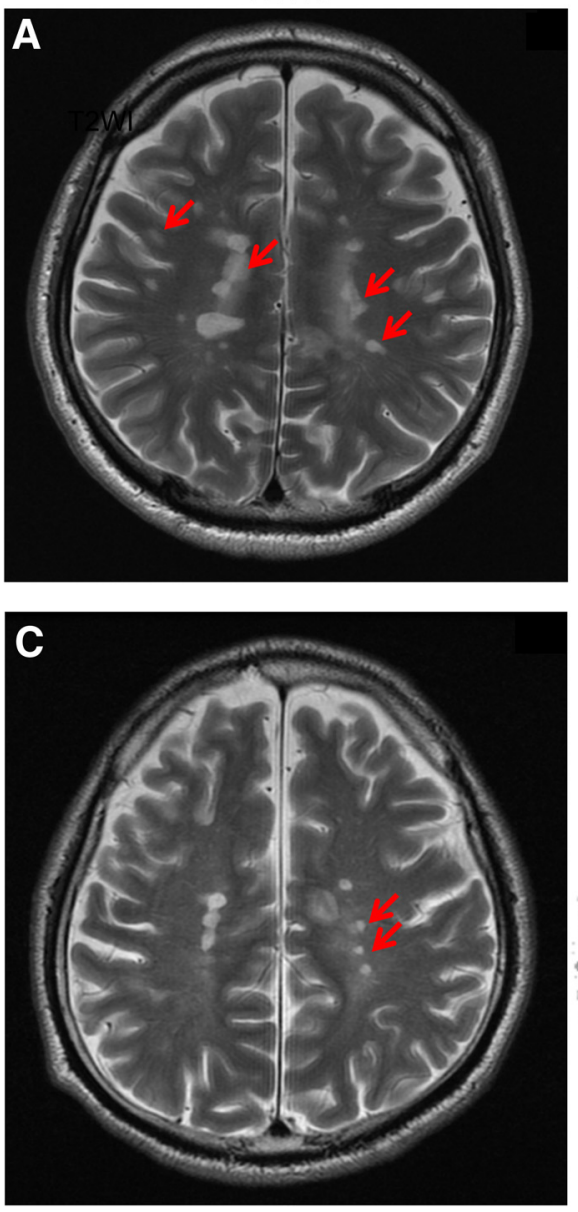
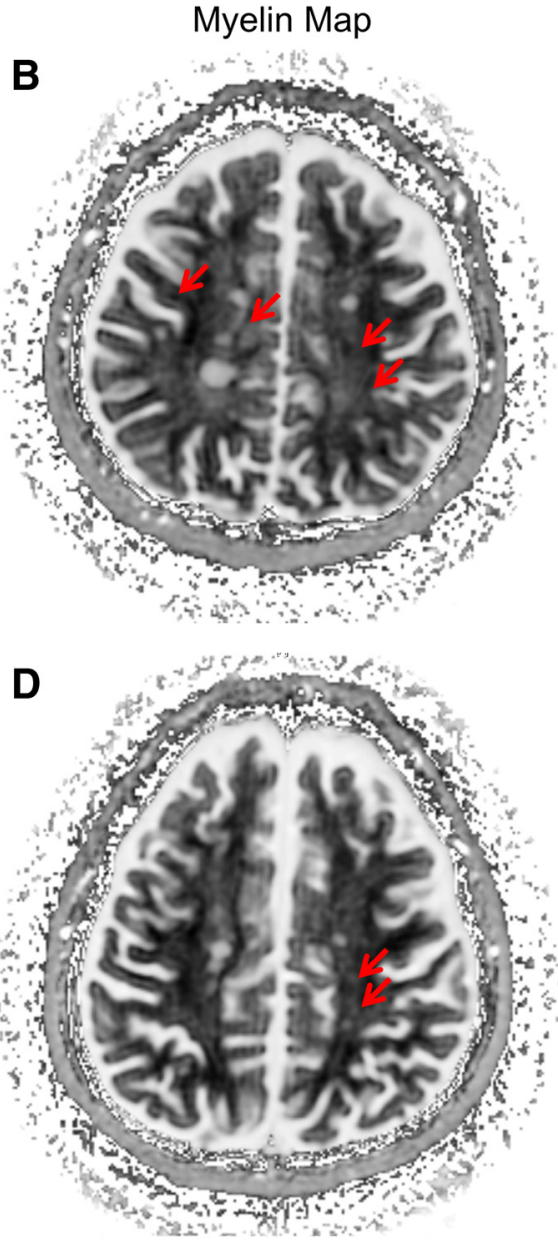

Figure 8. Myelin maps of MS patients. Axial T2WI and myelin map are shown for the brains of 2 patients: a relapsingremitting MS patient (patient \#1; 43-year-old male; $\boldsymbol{A}$ and $\boldsymbol{B}$, respectively) and a primary progressive MS patient (patient \#2; 40-year-old male; $($ and $\boldsymbol{D}$, respectively). Detailed clinical characteristics are summarized in Table 1. Red arrows indicate T2-hyperintense lesions with positive myelin signals in the myelin map (suggestive of possibly remyelinated lesions).

fact that T2WI was able to visualize lesions in the dysmyelinated spinal cord model (Fig. 2). These results also highlight the utility of the myelin map in detecting myelin signals both under dysmyelinating and demyelinating conditions.

\section{Myelin map of a normal human brain}

To verify the clinical utility of this new technique, we next acquired a myelin map of the normal human brain of a healthy volunteer (male, 40 years old). Using a $3 \mathrm{~T}$ MR scanner, the optimized QSI protocol required for the construction of the myelin map could be performed within $10 \mathrm{~min}$. The myelin map obtained by this protocol had a sufficient resolution to detect 

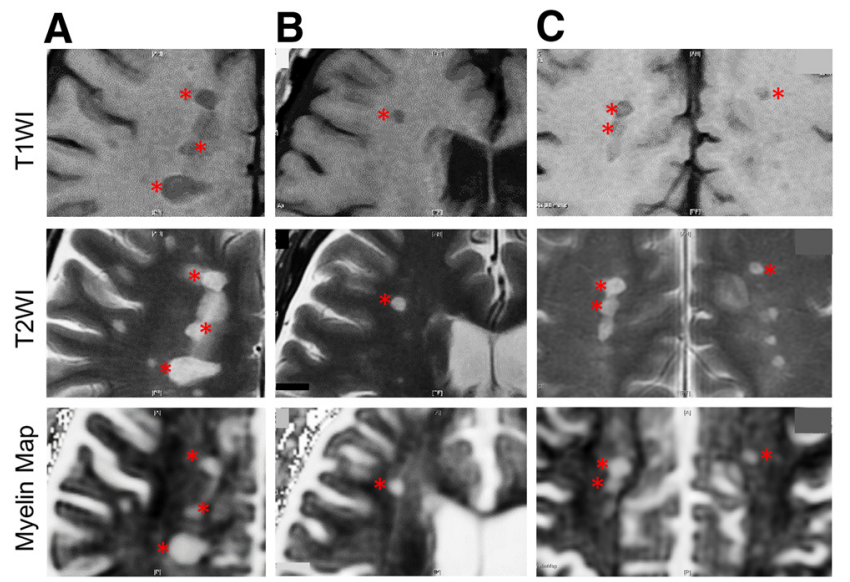

Figure 9. Myelin signals are virtually absent in T1 black holes. Axial T1WI, T2WI, and myelin maps are shown for the brains (deep cerebral white matter) of 2 patients: a relapsing-remitting MS patient (patient \#1; 43-year-old male; $\boldsymbol{A}$ and $\boldsymbol{B}$ ) and a primary progressive MS patient (patient \#2; 40-year-old male; $\boldsymbol{C}$ ). Red asterisks indicate T1hypointense lesions ( $\mathrm{T} 1$ black holes) where myelin signals are always absent in the myelin map. These data are consistent with those of a previous radiopathological correlation study (Barkhof et al., 2003).

myelinated tracts, including the cerebral peduncle in the midbrain and the pyramidal tracts in the medulla oblongata, where T2WI is often unable to differentiate myelinated fibers (Fig. 6). We also compared the relative contrast of the myelin map with that of the FA map and DTI maps. The myelin map showed a significantly higher contrast in detecting myelin signals, not only in deep white matter such as the corpus callosum, but also in cortical white matter (Fig. 7), suggesting that the myelin map is superior to the FA map or other DTI maps at detecting myelin signals in the human brain.

\section{Myelin map in MS patients}

Finally, we assessed the usefulness of the myelin map in a clinical setting by performing a pilot study in a selected group of MS patients.

The first patient was a 43-year-old male with a clinical diagnosis of relapsing-remitting MS (RRMS; see Table 1 for detailed clinical information). He had multiple, T2-hyperintense brain lesions that were compatible with an MS diagnosis. The myelin map revealed the presence of myelin signals in some of these lesions (Fig. $8 A, B$ ). The second patient was a 40-year-old male with a clinical diagnosis of primary progressive MS (PPMS; Table 1). Remyelination failure has been proposed as an explanation for the progressive clinical course of PPMS (Pender, 2004); however, a postmortem pathological study recently revealed the presence of remyelinated lesions in this condition (Bramow et al., 2010). The myelin map of this PPMS patient revealed myelin signals in some T2hyperintense lesions (Fig. 8C,D), consistent with the view that remyelination may occur in at least some PPMS patients. In both MS patients, myelin signals were virtually absent in T1hypointense lesions (also known as "T1 black holes"; Fig. 9). These findings are consistent with a previous histopathological study showing that T2-hyperintense lesions included both demyelinated and remyelinated lesions, whereas the majority of T1-hypointense lesions were demyelinated (Barkhof et al., 2003).

Finally, to assess the sensitivity of the myelin map in monitoring ongoing remyelination, we analyzed a 35-year-old female pa- tient with a clinical diagnosis of RRMS (Table 1) who had just suffered from an acute relapse that resulted in weakness and tactile hypoesthesia in her left arm. Her spinal MRI revealed a gadolinium-enhanced T2-hyperintense lesion in the left half of the cervical spinal cord at the C4/5 level (Fig. 10A). A diagnosis of MS relapse in the spinal cord was made and she was treated with intravenous methylprednisolone therapy (IVMP). Before IVMP, her disability score (expanded disability status scale; EDSS) (Kurtzke, 1983) was 2/10 and the myelin map showed an extensive loss of myelin signal at the same location as the hyperintense lesions on T2WI (Fig. 10B). Two weeks after IVMP, her weakness decreased and her EDSS score improved to 1.5/10 (Fig. 10C). At this time point, the myelin map detected the recovery of a weak myelin signal in the lesion, suggestive of remyelination. Ten weeks after IVMP, her symptoms completely resolved and her EDSS score was normal (0/10). The myelin map detected a significant reappearance of myelin signal in the lesion, which paralleled her clinical recovery (Fig. 10D). In contrast, the lesion remained hyperintense on T2WI throughout this time course, even when her clinical symptoms had resolved completely.

Collectively, these data suggest that the myelin map can be feasibly and reliably used in real-world settings to visualize myelin status in the CNS, including dysmyelination, demyelination, and possibly remyelination.

\section{Discussion}

In the present study, we introduced a novel modality that produces an image type termed the myelin map for the visualization of myelin status in the CNS. We first showed that the myelin map could be used successfully to visualize myelinated tracts in postmortem brains and spinal cords of mice (Figs. 2, 3). The myelin map of a live, nonhuman primate confirmed its high sensitivity in detecting demyelination and remyelination (Figs. 4, 5). A myelin map of the human brain could be obtained in $<10$ min using a 3 T MR scanner and was superior to conventional T2WI, FA map, and various DTI maps in depicting myelinated tracts (Figs. 6, 7). Finally, a pilot clinical study in MS patients suggested that the myelin map is useful for differentiating possibly remyelinated lesions from chronic demyelinated lesions (Figs. 8, 9). In addition, its sensitivity may enable clinicians to monitor the reappearance of myelin signals (i.e., possible remyelination activity) after an acute attack, which is beneficial because remyelination seems to parallel clinical recovery (Fig. 10).

Because myelin is constituted of hydrophobic lipid membranes, it restricts the free diffusion of water molecules rigorously. Therefore, the key principle for visualizing myelin by MRI is to calculate the parameters of water diffusion restriction. Previous studies have suggested that a PDF obtained with a high $b$-value, full-scale QSI allows calculation of the displacement distribution profile of water on the order of $1 \mu \mathrm{m}$ (King et al., 1994; Cohen et al., 2002). This high accuracy enables the differentiation of myelinated axons from nonmyelinated axons because the diameter of the former is usually $\sim 1-10 \mu \mathrm{m}$. The clinical application of QSI for the visualization of myelin status is, however, hindered by the amount of time required for the imaging. The quality of QSI depends on the range and steps of $b$-values applied, with higher accuracy resulting in more time-consuming scans. DKI was developed to reduce the time required for the imaging (Jensen et al., 2005) by estimating kurtosis values with only three low $b$-values, rather than precisely calculating the actual displacement distribution by full-scale QSI. DKI enables detection of microstructural changes during brain maturation and aging 
A

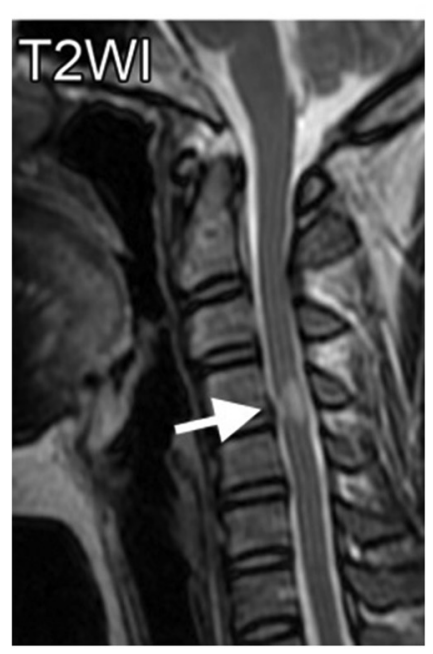

B
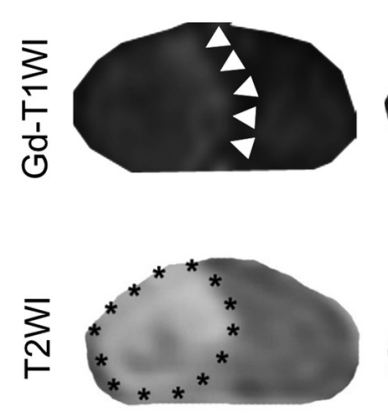

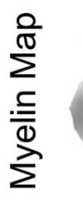

\section{Pre-treatment}

EDSS $=2.0$
C
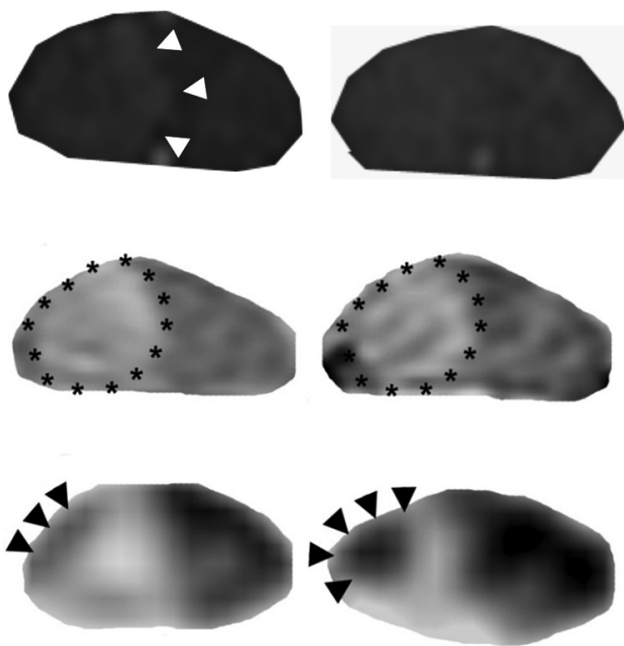
Post-treatment (2 weeks) EDSS $=1.5$

D

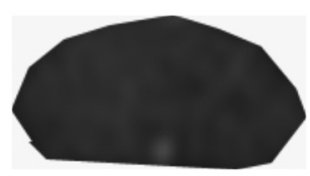

\section{Post-treatment \\ (10 weeks) \\ EDSS $=0$}

Figure 10. Sequential myelin maps during recovery after an attack in an MS patient. The figure shows a sagittal T2WI of the cervical spinal cord at onset of acute relapse in a relapsing-remitting MS patient (patient \#1; 43-year-old male) (A) and sequential axial T1-weighted images enhanced with gadolinium-based contrast agents (Gd-T1WI), T2WI, and myelin maps of the spinal cord at C4/5 at onset (pretreatment), 2 weeks after IVMP, and 10 weeks after IVMP (B, C, D, respectively). EDSS scores were 2.0, 1.5, and 0 at onset, 2 weeks after IVMP, and 10 weeks after IVMP, respectively. Abnormal gadolinium enhancement suggesting a breakdown of the blood-brain barrier is evident during the first 2 weeks after IVMP (white arrowheads). Although T2WI shows no significant differences throughout the study period (asterisks), the gadolinium enhancement disappears and the myelin map depicts the reappearance of myelin signals (black arrowheads, possibly reflecting remyelination), which correlated well with the clinical recovery.

(Falangola et al., 2008; Cheung et al., 2009), in gliomas (Raab et al., 2010), after brain injury (Grossman et al., 2012; Zhuo et al., 2012), in attention-deficit hyperactivity disorder (Helpern et al., 2011), and in cerebral infarction (Hori et al., 2012a). DKI may also serve as an indicator of tissue damage in normalappearing white matter in MS patients (Yoshida et al., 2013). Indeed, DKI is far less time consuming than full-scale QSI, although the diffusion resolution is decreased due to the omission of high $b$-values. This limitation is unavoidable in DKI because the diffusion-related signal decay curve in white matter is multiexponential (Cohen et al., 2002). Creation of the myelin map involved performing QSI with a limited number of high $b$-values to acquire kurtosis values with sufficient accuracy to differentiate the myelin status within white matter. Therefore, the principal difference between the myelin map and full-scale QSI is the number of $b$-value steps, whereas the difference between the myelin map and DKI is the range of $b$-values. In addition, the myelin map uses a heat map based on standardized kurtosis values (NLD), making it easier to compare data between individuals within the same species.

The major limitation of the current study is the lack of histological validation of the human myelin map, although the animal myelin map depicted the histologically verified myelin status accurately in mice and nonhuman primates. It has been reported that the diameter of axons in the corpus callosum is generally conserved across species (Olivares et al., 2001), supporting the view that the myelin map is valid in the human CNS as well. It is also important to note that the accuracy of the myelin map may be reduced in regions where biological diffusion barriers other than myelin exist, especially if they have relatively similar displacement distribution compared with that of the myelin surrounding axons. We were not able to confirm the presence of such barrier structures in our ex- periments, but this possibility should be taken into account, especially when applying the myelin map to various pathological conditions. In addition, kurtosis values may be influenced in part by other parameters such as membrane permeability and extracellular space fraction (Nilsson et al., 2013) or they may change with developmental stage (Cheung et al., 2009). The former possibility should be taken into account when applying the method to inflammatory neurological disorders such as MS and encephalitis. The myelin map should therefore be validated histologically in various human diseases in the future.

Recently, myelin has been gaining increasing attention in various neurological and psychiatric diseases. For example, myelin breakdown is hypothesized to be involved in the pathogenesis of Alzheimer's disease (Bartzokis, 2011), amyotrophic lateral sclerosis (Kang et al., 2013), and even schizophrenia (Tkachev et al., 2003; Mighdoll et al., 2015). The lack of methods to monitor CNS myelin has hindered the testing of this hypothesis; therefore, the myelin map may provide key clues for understanding these disorders. As for CNS demyelinating diseases, the myelin map may be useful for monitoring the effect of remyelination medicines currently under development (Mi et al., 2007) and for classifying patients according to remyelination capacity. The latter may be of particular importance in MS because the heterogeneous nature of remyelination capacity has been reported (Goldschmidt et al., 2009).

In summary, we successfully developed a modality that produces a novel image type, the myelin map, for the accurate imaging of CNS myelin that may enable clinicians to easily evaluate the state of myelination. The myelin map is obtained using 3 T MR scanners that are already available in the clinical setting and can therefore be immediately adapted for use. The 
myelin map promises to be a powerful tool for researchers and clinicians examining myelin-related diseases.

\section{References}

Assaf Y, Mayk A, Cohen Y (2000) Displacement imaging of spinal cord using $q$-space diffusion-weighted MRI. Magn Reson Med 44:713-722. Medline

Barkhof F (1999) MRI in multiple sclerosis: correlation with expanded disability status scale (EDSS). Mult Scler 5:283-286. Medline

Barkhof F, Bruck W, De Groot CJ, Bergers E, Hulshof S, Geurts J, Polman CH, van der Valk P (2003) Remyelinted lesions in multiple sclerosis: magnetic resonance image appearance. Arch Neurol 60:1073-1081. CrossRef Medline

Bartzokis G (2011) Alzheimer's disease as homeostatic responses to agerelated myelin breakdown. Neurobiol Aging 32:1341-1371. CrossRef Medline

Bramow S, Frischer JM, Lassmann H, Koch-Henriksen N, Lucchinetti CF, Sørensen PS, Laursen H (2010) Demyelination versus remyelination in progressive multiple sclerosis. Brain 133:2983-2998. CrossRef Medline

Câmara J, Wang Z, Nunes-Fonseca C, Friedman HC, Grove M, Sherman DL, Komiyama NH, Grant SG, Brophy PJ, Peterson A, ffrenchConstant C (2009) Integrin-mediated axoglial interactions initiate myelination in the central nervous system. J Cell Biol 185:699-712. CrossRef Medline

Cheung MM, Hui ES, Chan KC, Helpern JA, Qi L, Wu EX (2009) Does diffusion kurtosis imaging lead to better neural tissue characterization? A rodent brain maturation study. Neuroimage 45:386-392. CrossRef Medline

Cohen Y, Assaf Y (2002) High $b$-value $q$-space analyzed diffusion-weighted MRS and MRI in neuronal tissues-a technical review. NMR Biomed 15: 516-542. CrossRef Medline

Engelbrecht V, Scherer A, Rassek M, Witsack HJ, Mödder U (2002) Diffusion-weighted MR imaging in the brain in children: findings in the normal brain and in the brain with white matter diseases. Radiology 222: 410-418. CrossRef Medline

Falangola MF, Jensen JH, Babb JS, Hu C, Castellanos FX, Di Martino A, Ferris SH, Helpern JA (2008) Age-related non-Gaussian diffusion patterns in the prefrontal brain. J Magn Reson Imaging 28:1345-1350. CrossRef Medline

Gass A, Barker GJ, Kidd D, Thorpe JW, MacManus D, Brennan A, Tofts PS, Thompson AJ, McDonald WI, Miller DH (1994) Correlation of magnetization transfer ratio with clinical disability in multiple sclerosis. Ann Neurol 36:62-67. CrossRef Medline

Goldschmidt T, Antel J, König FB, Brück W, Kuhlmann T (2009) Remyelination capacity of the MS brain decreases with disease chronicity. Neurology 72:1914-1921. CrossRef Medline

Grossman EJ, Ge Y, Jensen JH, Babb JS, Miles L, Reaume J, Silver JM, Grossman RI, Inglese M (2012) Thalamus and cognitive impairment in mild traumatic brain injury: a diffusional kurtosis imaging study. J Neurotrauma 29:2318-2327. CrossRef Medline

Hall SM (1972) The effect of injections of lysophosphatidyl choline into white matter of the adult mouse spinal cord. J Cell Sci 10:535-546. Medline

Hamazaki N, Sato S, Hori M, Fukunaga I, Kosaka T, Ito K, Suzuki Y, Houshito H, Masutani Y, Aoki S (2012) Optimization for q-space imaging in a clinical setting: setting the q-value. Jpn J Magn Res Med Sci 32:85-93.

Helpern JA, Adisetiyo V, Falangola MF, Hu C, Di Martino A, Williams K, Castellanos FX, Jensen JH (2011) Preliminary evidence of altered gray and white matter microstructural development in the frontal lobe of adolescents with attention -deficit hyperactivity disorder: a diffusional kurtosi imaging study. J Magn Reson Imaging 33:17-23. CrossRef Medline

Hori M, Aoki S, Fukunaka I, Suzuki Y, Masutani Y (2012a) A new diffusion metric, diffusion kurtosis imaging, used in the serial examination of a patient with stroke. Acta Radiol Short Rep 1:pii: arsr.2011.110024. CrossRef Medline

Hori M, Fukunaga I, Masutani Y, Taoka T, Kamagata K, Suzuki Y, Aoki S (2012b) Visualizing non-Gaussian diffusion: clinical application of $q$-space imaging and diffusional kurtosis imaging of the brain and spine. Magn Reson Med Sci 11:221-233. CrossRef Medline
Jensen JH, Helpern JA, Ramani A, Lu H, Kaczynski K (2005) Diffusional kurtosis imaging: the quantification of non-Gaussian water diffusion by means of magnetic resonance imaging. Magn Reson Med 53:1432-1440. CrossRef Medline

Kang SH, Li Y, Fukaya M, Lorenzini I, Cleveland DW, Ostrow LW, Rothstein JD, Bergles DE (2013) Degeneration and impaired regeneration of gray matter oligodendrocytes in amyotrophic lateral sclerosis. Nat Neurosci 16:571-579. CrossRef Medline

Kimura M, Inoko H, Katsuki M, Ando A, Sato T, Hirose T, Takashima H, Inayama S, Okano H, Takamatsu K (1985) Molecular genetic analysis of myelin-deficient mice: shiverer mutant mice show deletion in gene(s) coding for myelin basic protein. J Neurochem 44:692-696. CrossRef Medline

King MD, Houseman J, Roussel SA, van Bruggen N, Williams SR, Gadian DG (1994) $q$-Space imaging of the brain. Magn Reson Med 32:707-713. CrossRef Medline

KurtzkeJF (1983) Rating neurologic impairment in multiple sclerosis: an expanded disability status scale (EDSS). Neurology 33:1444-1452. CrossRef Medline

Laule C, Vavasour IM, Moore GR, Oger J, Li DK, Paty DW, MacKay AL (2004) Water content and myelin water fraction in multiple sclerosis. A T2 relaxation study. J Neurol 251:284-293. CrossRef Medline

Lee Y, Morrison BM, Li Y, Lengacher S, Farah MH, Hoffman PN, Liu Y, Tsingalia A, Jin L, Zhang PW, Pellerin L, Magistretti PJ, Rothstein JD (2012) Oligodendroglia metabolically support axons and contribute to neurodegeneration. Nature 487:443-448. CrossRef Medline

Liu J, Dietz K, DeLoyht JM, Pedre X, Kelkar D, Kaur J, Vialou V, Lobo MK, Dietz DM, Nestler EJ, Dupree J, Casaccia P (2012) Impaired adult myelination in the prefrontal cortex of socially isolated mice. Nat Neurosci 15:1621-1623. CrossRef Medline

Marcus J, Honigbaum S, Shroff S, Honke K, Rosenbluth J, Dupree JL (2006) Sulfatide is essential for the maintenance of CNS myelin and axon structure. Glia 53:372-381. CrossRef Medline

Mi S, Hu B, Hahm K, Luo Y, Kam Hui ES, Yuan Q, Wong WM, Wang L, Su H, Chu TH, Guo J, Zhang W, So KF, Pepinsky B, Shao Z, Graff C, Garber E, Jung V, Wu EX, Wu W (2007) LINGO-1 antagonist promotes spinal cord remyelination and axonal integrity in MOGinduced experimental autoimmune encephalomyelitis. Nat Med 13: 1228-1233. CrossRef Medline

Mighdoll MI, Tao R, Kleinman JE, Hyde TM (2015) Myelin, myelin-related disorders, and psychosis. Schizophr Res 161:85-93. CrossRef Medline

Nave KA, Bloom FE, Milner RJ (1987) A single nucleotide difference in the gene for myelin proteolipid protein defines the jimpy mutation in mouse. J Neurochem 49:1873-1877. CrossRef Medline

Nilsson M, van Westen D, Ståhlberg F, Sundgren PC, LättJ (2013) The role of tissue microstructure and water exchange in biophysical modelling of diffusion in white matter. MAGMA 26:345-370. CrossRef Medline

Nossin-Manor R, Duvdevani R, Cohen Y (2002) $q$-space high $b$ value diffusion MRI of hemi-crush in rat spinal cord: evidence for spontaneous regeneration. Magn Reson Imaging 20:231-241. CrossRef Medline

Okano H, Miura M, Moriguchi A, Ikenaka K, Tsukada Y, Mikoshiba K (1987) Inefficient transcription of the myelin basic protein gene possibly causes hypomyelination in myelin-deficient mutant mice. J Neurochem 48:470-476. CrossRef Medline

Olivares R, Montiel J, Aboitiz F (2001) Species differences and similarities in the fine structure of the mammalian corpus callosum. Brain Behav Evol 57:98-105. CrossRef Medline

Pender MP (2004) The pathogenesis of primary progressive multiple sclerosis: antibody-mediated attack and no repair? J Clin Neurosci 11:689692. CrossRef Medline

Pfefferbaum A, Sullivan EV, Hedehus M, Lim KO, Adalsteinsson E, Moseley M (2000) Age-related decline in brain white matter anisotropy measured with spatially corrected echo-planar diffusion tensor imaging. Magn Reson Med 44:259-268. CrossRef Medline

Raab P, Hattingen E, Franz K, Zanella FE, Lanfermann H (2010) Cerebral gliomas: diffusional kurtosis imaging analysis of microstructural differences. Radiology 254:876-881. CrossRef Medline

Shibata S, Murota Y, Nishimoto Y, Yoshimura M, Nagai T, Okano H, Siomi MC (2015) Immuno-electron microscopy and electron microscopic in situ hybridization for visualizing piRNA biogenesis bodies in drosophila ovaries. Methods Mol Biol 1328:163-178. CrossRef Medline

Stankoff B, Freeman L, Aigrot MS, Chardain A, Dollé F, Williams A, Galanaud D, Armand L, Lehericy S, Lubetzki C, Zalc B, Bottlaender M (2011) Imaging central nervous system myelin by positron emission 
tomography in multiple sclerosis using [methyl- $\left.{ }^{11} \mathrm{C}\right]-2-\left(4^{\prime}\right.$-methylaminophenyl)-6-hydroxybenzothiazole. Ann Neurol 69:673-680. CrossRef Medline

Stejskal EO, Tanner JE (1965) Spin diffusion measurements: spin echoes in the presence of a time dependent field gradient. J Chem Phys 42:288 -292. CrossRef

Tkachev D, Mimmack ML, Ryan MM, Wayland M, Freeman T, Jones PB, Starkey M, Webster MJ, Yolken RH, Bahn S (2003) Oligodendrocyte dysfunction in schizophrenia and bipolar disorder. Lancet 362:798-805. CrossRef Medline

Watanabe M, Toyama Y, Nishiyama A (2002) Differentiation of prolifer- ated NG2-positive glial progenitor cells in a remyelinating lesion. J Neurosci Res 69:826-836. CrossRef Medline

Yoshida M, Hori M, Yokoyama K, Fukunaga I, Suzuki M, Kamagata K, Shimoji K, Nakanishi A, Hattori N, Masutani Y, Aoki S (2012) Diffusional kurtosis imaging of normal-appearing white matter in multiple sclerosis: preliminary clinical experience. Jpn J Radiol 31:50-55. Medline

Zhuo J, Xu S, Proctor JL, Mullins RJ, Simon JZ, Fiskum G, Gullapalli RP (2012) Diffusion kurtosis as an in vivo imaging marker for reactive gliosis in traumatic brain injury. Neuroimage 59:467-477. CrossRef Medline 